\title{
Existence of Solutions of Periodic Boundary Value Problems for Impulsive Functional Duffing Equations at Nonresonance Case
}

\author{
Xingyuan Liu' ${ }^{1}$ and Yuji Liu ${ }^{2}$ \\ ${ }^{1}$ Department of Mathematics, Shaoyang University, Shaoyang, Hunan 422000, China \\ ${ }^{2}$ Department of Mathematics, Guangdong University of Business Studies, Guangzhou, \\ Guangdong 510320, China
}

Correspondence should be addressed to Xingyuan Liu, liuxingyuan999@sohu.com

Received 19 June 2008; Accepted 27 August 2008

Recommended by Zhitao Zhang

This paper deals with the existence of solutions of the periodic boundary value problem of the impulsive Duffing equations: $x^{\prime \prime}(t)+\alpha x^{\prime}(t)+\beta x(t)=f\left(t, x(t), x\left(\alpha_{1}(t)\right), \ldots, x\left(\alpha_{n}(t)\right)\right)$, a.e. $t \in$ $[0, T], \Delta x\left(t_{k}\right)=I_{k}\left(x\left(t_{k}\right), x^{\prime}\left(t_{k}\right)\right), k=1, \ldots, m, \Delta x^{\prime}\left(t_{k}\right)=J_{k}\left(x\left(t_{k}\right), x^{\prime}\left(t_{k}\right)\right), k=1, \ldots, m, x^{(i)}(0)=$ $x^{(i)}(T), i=0,1$. Sufficient conditions are established for the existence of at least one solution of above-mentioned boundary value problem. Our method is based upon Schaeffer's fixed-point theorem. Examples are presented to illustrate the efficiency of the obtained results.

Copyright (C) 2008 X. Liu and Y. Liu. This is an open access article distributed under the Creative Commons Attribution License, which permits unrestricted use, distribution, and reproduction in any medium, provided the original work is properly cited.

\section{Introduction}

In recent years, many authors studied the solvability of the periodic boundary value problems (PBVPs for short) for second-order ordinary or functional differential equations with or without impulse effects; see [1-24] and the references therein. For example, consider the following PBVP:

$$
\begin{gathered}
x^{\prime \prime}(t)=f(t, x(t)), \quad t \in(0,2 \pi), \\
x(0)=x(2 \pi), \quad x^{\prime}(0)=x^{\prime}(2 \pi),
\end{gathered}
$$

the well-known result is that if $f$ satisfies the nonresonance condition

$$
-(N+1)^{2}+\epsilon \leq f_{u}(t, u) \leq-\epsilon-N^{2},
$$

where $N$ is a nonnegative integer and $\epsilon$ is a positive constant, then $\operatorname{PBVP}(1.1)$ has a unique solution; see [1]. 
For PBVP of the Duffing equation

$$
\begin{gathered}
x^{\prime \prime}(t)+c x^{\prime}(t)+g(t, x(t))=e(t), \quad t \in(0,2 \pi), \\
x(0)=x(2 \pi), \quad x^{\prime}(0)=x^{\prime}(2 \pi),
\end{gathered}
$$

in [16], the authors proved the following results.

Theorem 1.1. Suppose $g$ is a $L^{2}$-Caratheodory function, and there are $a \leq A, r<0<R$ such that

$$
g(t, x) \geq A, \quad x \geq R, t \in[0,2 \pi], \quad g(t, x) \leq a, \quad x \leq r, t \in[0,2 \pi],
$$

and further there is $r \in L(0,2 \pi)$ with $\|r\|_{\infty}<1+C^{2}$ such that

$$
\varlimsup_{|x| \rightarrow \infty} \frac{g(t, x)}{x}<r(t), \quad t \in(0,2 \pi) .
$$

Then, $P B V P(1.3)$ has at least one solution for each $e \in L^{2}(0,2 \pi)$ with $a \leq(1 / 2 \pi) \int_{0}^{2 \pi} e(s) d s \leq A$.

In [17], Nieto and Rodríguez-López studied the following PBVP:

$$
\begin{gathered}
x^{\prime \prime}(t)+a x^{\prime}(t)+b x(t)+c x^{\prime}([t])+d x([t])=\sigma(t), \quad t \in(0, T), \\
x(0)=x(T), \quad x^{\prime}(0)=x^{\prime}(T) .
\end{gathered}
$$

They gave Green's function to express the unique solution for the correspondence secondorder functional differential equation with periodic boundary conditions and the functional dependence given by the piecewise constant function. Using upper and lower solution methods, they presented sufficient conditions to assure the existence of solutions of $\operatorname{PBVP}(1.6)$. The authors in $[3,21]$ also studied the solvability of $\operatorname{PBVP}(1.6)$ by the similar method.

In [2], Ding et al. studied the PBVP:

$$
\begin{gathered}
x^{\prime \prime}(t)+f(t, x(t), x(\theta(t)))=0, \quad t \in(0, T), \\
x(0)=x(T), \quad x^{\prime}(0)=x^{\prime}(T) .
\end{gathered}
$$

Sufficient conditions for the existence of solutions of PBVP(1.7) are given by using upper and lower solution method.

The PBVPs,

$$
\begin{gathered}
x^{\prime \prime}(t)+\rho^{2} x(t)=f(t, x(t)), \quad t \in(0,2 \pi), \\
x(0)=x(2 \pi), \quad x^{\prime}(0)=x^{\prime}(2 \pi), \\
-x^{\prime \prime}(t)+\rho^{2} x(t)=f(t, x(t)), \quad t \in(0,2 \pi), \\
x(0)=x(2 \pi), \quad x^{\prime}(0)=x^{\prime}(2 \pi),
\end{gathered}
$$

were studied in [8, 18-20]. In [19], based upon Krasnosel'skii fixed-point theorems, Jiang proved that $\mathrm{PBVP}(1.8)$ and $\mathrm{PBVP}(1.9)$ with singularity have at least one positive solution provided that $f(t, x)$ is superlinear or sublinear at $x=0$ and $x=+\infty$. In [21], Wang, who utilized the Schauder fixed-point theorem, proved that PBVP(1.9) without singularity has at 
least one positive solution provided that $f(t, x)$ is sublinear at $x=\infty$. In [18], Zhang and Wang established multiplicity results to positive solutions of $\operatorname{PBVP}(1.8)$ and $\operatorname{PBVP}(1.9)$ with $f$ being a Caratheodory function.

In paper [24], Liu and Ge studied the following equation:

$$
x^{\prime \prime}(t)-p(t) x^{\prime}(t)+q(t) x(t)=\lambda f(t, x(t-\tau(t)))+r(t) ;
$$

they established sufficient conditions for the existence of positive periodic solutions of (1.10). equation:

In [11], Peng studied the existence of periodic solutions of the functional Duffing

$$
x^{\prime \prime}(t)+c x^{\prime}(t)+g_{1}\left(t, x\left(t-\tau_{1}(t)\right)\right)+g_{2}\left(t, x\left(t-\tau_{2}(t)\right)\right)=p(t)
$$

The studies on the existence of solutions of the periodic boundary value problems of the second-order impulsive differential equations,

$$
\begin{gathered}
x^{\prime \prime}(t)+f(t, x(t))=0, \quad t \in(0, T), \\
x(0)=x(T), \quad x^{\prime}(0)=x^{\prime}(T), \\
\Delta x^{(i)}\left(t_{k}\right)=I_{i, k}\left(x\left(t_{k}\right)\right), \quad k=1, \ldots, p, i=0,1,
\end{gathered}
$$

can be found in $[3,4,12-14,20,22,23]$ and the references therein. The methods used there are of lower and upper solutions methods, the monotone iterative technique.

In all above-mentioned papers, the results are based upon the following assumptions.

(H) $f$ satisfies either the Lipschitzian condition, left Lipschitzian condition, right Lipschitzian condition, or Nagumo conditions.

To the best of our knowledge, the existence of solutions of periodic boundary value problems for impulsive Duffing functional differential equations has not been well studied till now.

In this paper, we investigate the following periodic boundary value problem for the impulsive functional Duffing equation:

$$
\begin{aligned}
& x^{\prime \prime}(t)+\alpha x^{\prime}(t)+\beta x(t)=f\left(t, x(t), x\left(\alpha_{1}(t)\right), \ldots, x\left(\alpha_{n}(t)\right)\right), \quad \text { a.e. } t \in[0, T], \\
& \Delta x\left(t_{k}\right)=I_{k}\left(x\left(t_{k}\right), x^{\prime}\left(t_{k}\right)\right), \quad k=1, \ldots, m, \\
& \Delta x^{\prime}\left(t_{k}\right)=J_{k}\left(x\left(t_{k}\right), x^{\prime}\left(t_{k}\right)\right), \quad k=1, \ldots, m, \\
& x^{(i)}(0)=x^{(i)}(T), \quad i=0,1,
\end{aligned}
$$

where $m$ is a positive integer, $\alpha, \beta \in R, f:[0, T] \times R^{n+1} \rightarrow R$ is a impulsive Caratheodory function, $\alpha_{i} \in C^{1}([0, T],[0, T])$, whose inverse function is denoted by $\beta_{i}$ with $\beta_{i} \in C^{1}[0, T]$, $I_{k}, J_{k}: R^{2} \rightarrow R$ are continuous.

Our purpose here is to provide sufficient conditions for the existence of solutions of (1.13) at nonresonance case. This will be done by applying the well-known Schaeffer's fixedpoint theorem, and we do not rely on the existence of upper and lower solutions and the assumption (H) mentioned above. 
The main results and examples in this paper are established in Section 2. The proofs of the main results are presented in Section 3.

\section{Main results and examples}

In this section, we establish the main results and examples to illustrate the main theorems. To define solutions of PBVP (1.13), we introduce the following Banach spaces and definitions.

Suppose $u: J=[0, T] \rightarrow R$, and $0=t_{0}<t_{1}<\cdots<t_{m}<t_{m+1}=T$. For $k=0, \ldots, m$, define the function $u_{k}:\left(t_{k}, t_{k+1}\right) \rightarrow R$ by $u_{k}(t)=u(t)$. Choose

$$
X=\left\{u: J \rightarrow R \mid u_{k} \in C^{0}\left(t_{k}, t_{k+1}\right), k=0, \ldots, m, \text { there exist the limits } \begin{array}{r}
\lim _{t \rightarrow t_{k}^{-}} u(t)=u\left(t_{k}\right), \\
\lim _{t \rightarrow 0^{+}} u(t), \\
\lim _{t \rightarrow T^{-}} u(t)=u(T)
\end{array}\right\}
$$

with the norm

$$
\|u\|_{X}=\|u\|=\sup _{t \in[0, T]}|u(t)|
$$

for $u \in X$. Choose

$$
Y=X \times R^{m} \times R^{m}
$$

with the norm

$$
\|y\|_{Y}=\|y\|=\max \left\{\sup _{t \in[0, T]}|u(t)|, \max _{1 \leq k \leq m}\left\{\left|x_{k}\right|\right\}, \max _{1 \leq k \leq m}\left|y_{k}\right|\right\}
$$

for $y=\left\{u, x_{1}, \ldots, x_{m}, y_{1}, \ldots, y_{m}\right\} \in Y$. Then, $X$ and $Y$ are real Banach spaces.

A function $F:[0,1] \times R^{n+1} \rightarrow R$ is called an impulsive Caratheodory function if

(i) $F\left(\bullet, u_{0}, u_{1}, \ldots, u_{n}\right) \in X$ for each $u=\left(u_{0}, \ldots, u_{n}\right) \in R^{n+1}$;

(ii) $F(t, \bullet, \ldots, \bullet)$ is continuous for $t \neq t_{k}(k=1, \ldots, m)$. conditions:

By a solution of PBVP (1.13), we mean a function $x:[0, T] \rightarrow R$ satisfying the following

(i) $x \in X$ is differentiable in $\left(t_{k}, t_{k+1}\right)(k=0,1, \ldots, m)$, there exist the limits $\lim _{t \rightarrow t_{k}^{+}} x^{\prime}(t), \lim _{t \rightarrow t_{k}^{-}} x^{\prime}(t)=x^{\prime}\left(t_{k}\right)(k=0,1, \ldots, m), \lim _{t \rightarrow 0^{+}} x^{\prime}(t)=x^{\prime}(0)$ and $\lim _{t \rightarrow T^{-}} x^{\prime}(t)=x^{\prime}(T)$;

(ii) $x^{\prime} \in X$ is differentiable in $\left(t_{k}, t_{k+1}\right)(k=0,1, \ldots, m)$, there exist the limits $\lim _{t \rightarrow t_{k}^{+}} x^{\prime \prime}(t), \lim _{t \rightarrow t_{k}^{-}} x^{\prime \prime}(t)=x^{\prime \prime}\left(t_{k}\right)(k=0,1, \ldots, m), \lim _{t \rightarrow 0^{+}} x^{\prime \prime}(t)=x^{\prime \prime}(0)$, and $\lim _{t \rightarrow T^{-}} x^{\prime \prime}(t)=x^{\prime \prime}(T)^{2}$

(iii) $x^{\prime \prime} \in X$;

(iv) the equations in (1.13) are satisfied. 
Consider the following homogenous PBVP:

$$
\begin{gathered}
x^{\prime \prime}(t)+\alpha x^{\prime}(t)+\beta x(t)=0, \quad t \in[0, T], \\
\Delta x\left(t_{k}\right)=0, \quad k=1, \ldots, m, \\
\Delta x^{\prime}\left(t_{k}\right)=0, \quad k=1, \ldots, m, \\
x(0)=x(T), \quad x^{\prime}(0)=x^{\prime}(T) .
\end{gathered}
$$

If $x \in X$ is a solution of problem (2.5), then

$$
\begin{aligned}
& x^{\prime \prime}(t) x(t)+\alpha x^{\prime}(t) x(t)+\beta[x(t)]^{2}=0 \\
& x^{\prime \prime}(t) x^{\prime}(t)+\alpha\left[x^{\prime}(t)\right]^{2}+\beta x(t) x^{\prime}(t)=0 .
\end{aligned}
$$

Integrating them from 0 to $T$, one sees that

$$
-\int_{0}^{T}\left[x^{\prime}(t)\right]^{2} d t+\beta \int_{0}^{T}[x(t)]^{2} d t=0, \quad \alpha \int_{0}^{T}\left[x^{\prime}(t)\right]^{2} d t=0 .
$$

If $\beta>0$ and $\alpha \neq 0$ or $\beta<0$, we get $x(t) \equiv 0$, then problem (2.5) has unique solution $x(t)=0$ at the cases either $\beta>0$ and $\alpha \neq 0$ or $\beta<0$. We call PBVP (1.13) at nonresonance case. It suffices to consider the following cases:

$$
\begin{aligned}
& \text { Case 1. } \alpha \geq 0, \beta<0, \quad \text { Case 2. } \alpha<0, \beta<0, \\
& \text { Case 3. } \alpha>0, \beta>0, \quad \text { Case 4. } \alpha<0, \beta>0 .
\end{aligned}
$$

We set the following assumptions which should be used in the main results.

$\left(A_{1}\right) x I_{k}(x, y) \geq 0$ for all $x, y \in R$.

$\left(A_{1}^{\prime}\right)\left[I_{k}(x, y)\right]^{2}+x I_{k}(x, y) \leq 0$ for all $x, y \in R$.

$\left(A_{2}\right) x J_{k}(x, y)+y I_{k}(x, y)+\lambda I_{k}(x, y) J_{k}(x, y) \geq 0$ for all $x, y \in R, \lambda \in[0,1]$.

$\left(A_{2}^{\prime}\right) x J_{k}(x, y)+y I_{k}(x, y)+\lambda I_{k}(x, y) J_{k}(x, y) \leq 0$ for all $x, y \in R, \lambda \in[0,1]$.

$\left(A_{3}\right)$ There exist constants $\theta_{k} \geq 0$ such that $\left|I_{k}(x, y)\right| \leq \theta_{k}|x|$ for all $x, y \in R$ with $\sum_{k=1}^{m} \theta_{k}<1$.

(C) There exist impulsive Caratheodory functions $h:[0, T] \times R^{n} \rightarrow R, g_{i}:[0, T] \times R \rightarrow R$, and function $r \in X$ and such that

(i) $f\left(t, x_{0}, \ldots, x_{n}\right)=h\left(t, x_{0}, \ldots, x_{n}\right)+\sum_{i=0}^{n} g_{i}\left(t, x_{i}\right)+r(t)$ holds for all $\left(t, x_{0}, \ldots, x_{n}\right) \in$ $[0, T] \times R^{n+1}$.

(ii) There exist constants $q \geq 0$ and $\theta>0$ such that

$$
h\left(t, x_{0}, \ldots, x_{n}\right) x_{0} \geq \theta\left|x_{0}\right|^{q+1}
$$

holds for all $\left(t, x_{0}, \ldots, x_{n}\right) \in[0, T] \times R^{n+1}$.

(iii) $\lim _{|x| \rightarrow+\infty} \sup _{t \in[0, T]}\left(\left|g_{i}(t, x)\right| /|x|^{q}\right)=r_{i} \in[0,+\infty)$ for $i=0, \ldots, n$.

$\left(C^{\prime}\right)$ There exist impulsive Caratheodory functions $h:[0, T] \times R^{n} \rightarrow R, g_{i}:[0, T] \times R \rightarrow R$, and function $r \in X$ and such that $(C)$ (i) and (C) (iii) hold. 
(ii) There exist constants $q \geq 0$ and $\theta>0$ such that

$$
h\left(t, x_{0}, \ldots, x_{n}\right) x_{0} \leq-\theta\left|x_{0}\right|^{q+1}
$$

holds for all $\left(t, x_{0}, \ldots, x_{n}\right) \in[0, T] \times R^{n+1}$.

Theorem 2.1. Suppose $\alpha \geq 0$ and $\beta<0,\left(A_{1}\right),\left(A_{2}\right),\left(A_{3}\right)$, and $(C)$ hold. Then, PBVP (1.13) has at least one solution if

$$
\theta>r_{0}+\sum_{k=1}^{n} r_{k}\left\|\beta_{k}^{\prime}\right\|^{q /(q+1)}
$$

holds.

Theorem 2.2. Suppose $\alpha<0$ and $\beta<0,\left(A_{1}^{\prime}\right),\left(A_{2}\right),\left(A_{3}\right)$, and $(C)$ hold. Then, PBVP (1.13) has at least one solution if (2.11) holds.

Theorem 2.3. Suppose $\alpha>0$ and $\beta>0,\left(A_{1}\right),\left(A_{2}\right),\left(A_{3}\right)$, and $(C)$ hold. Then, PBVP (1.13) has at least one solution if (2.11) holds.

Theorem 2.4. Suppose $\alpha<0$ and $\beta>0,\left(A_{1}\right),\left(A_{2}\right),\left(A_{3}\right)$, and $(C)$ hold. Then, PBVP (1.13) has at least one solution if (2.11) holds.

To illustrate our main results, we present two boundary value problems that our results can readily apply, whereas the known results in the current literature do not cover.

Example 2.5. Consider the following PBVP:

$$
\begin{gathered}
x^{\prime \prime}(t)+\alpha x^{\prime}(t)+\beta x(t)=\sum_{k=0}^{2 q+1} \epsilon_{k} x^{k}(t)+r(t), \quad t \in[0, T], t \neq t_{k}, k=1, \ldots, m, \\
\Delta x\left(t_{k}\right)=0, \quad k=1, \ldots, m, \\
\Delta x^{\prime}\left(t_{k}\right)=b_{k}\left[x\left(t_{k}\right)\right]^{3}, \quad k=1, \ldots, m, \\
x(0)=x(T), \quad x^{\prime}(0)=x^{\prime}(T),
\end{gathered}
$$

where $q$ is a positive integer, $\epsilon_{k} \geq 0, T>0, r$ is a continuous function. Corresponding to PBVP (1.13), one finds that

$$
\begin{gathered}
f\left(t, x_{0}, x_{1}, \ldots, x_{n}\right)=\sum_{k=0}^{2 q+1} \epsilon_{k} x_{0}^{k}+r(t), \\
I_{k}(x, y)=0, \quad J_{k}(x, y)=b_{k} x^{3},
\end{gathered}
$$

It is easy to see that

(i) $\left(A_{1}\right)$ holds,

(ii) since $a_{k} \geq 0$, one gets that $y I_{k}(x, y)+x J_{k}(x, y)+\lambda I_{k}(x, y) J_{k}(x, y)=b_{k} x^{4} \geq 0$, then $\left(A_{2}\right)$ holds,

(iii) $\left(A_{3}\right)$ holds, 
(iv) $h(t, x)=\epsilon_{2 q+1} x^{2 q+1}$ with $\alpha_{2 q+1}>0$ and $g_{k}(t, x)=\alpha_{k} x^{k}$ for $k=0,1, \ldots, 2 q$ implies that $(C)$ holds.

It follows from Theorem 2.1 that PBVP (2.12) has at least one solution.

Example 2.6. Consider the following PBVP:

$$
\begin{aligned}
x^{\prime \prime}(t)+\alpha x^{\prime}(t)+\beta x(t) & =\sum_{k=0}^{2 q+1} \alpha_{k} x^{k}(t)+\sum_{k=1}^{n} \beta_{k} x^{2 q+1}\left(\frac{1}{n+1} t\right)+r(t), \\
t \in[0, T], t \neq t_{k}, k=1, \ldots, m, & \\
\Delta x & \left(t_{k}\right)=0, \quad k=1, \ldots, m, \\
\Delta x^{\prime}\left(t_{k}\right) & =b_{k}\left[x\left(t_{k}\right)\right]^{5}, \quad k=1, \ldots, m, \\
x(0) & =x(T), \quad x^{\prime}(0)=x^{\prime}(T),
\end{aligned}
$$

where $\alpha \geq 0, \beta<0, T>0, q$ is a positive integer, $\alpha_{2 q+1}>0, m, n$ are positive integers, $r \in L^{1}[0, T], b_{k} \in R, 0<t_{1}<\cdots<t_{m}<T, \alpha_{k}, \beta_{k} \in R$.

It is easy to see that

(i) $\left(A_{1}^{\prime}\right)$ holds,

(ii) It is easy to see that

$$
x J_{k}(x, y)+y I_{k}(x, y)+\lambda I_{k}(x, y) J_{k}(x, y)=b_{k} x^{6} \geq 0
$$

if $b_{k} \geq 0$, then $\left(A_{2}\right)$ holds,

(iii) $\left(A_{3}\right)$ holds,

(iv) if $h(t, x)=\alpha_{2 q+1} x^{2 q+1}$ with $\alpha_{2 q+1}>0$ and $g_{k}(t, x)=\beta_{k} x^{2 q+1}$ for $k=1, \ldots, n, g_{0}(t, x)=$ $\sum_{k=0}^{2 q} \alpha_{k} x^{k}$, then $(C)$ holds.

It follows from Theorem 2.2 that PBVP (2.14) has at least one solution if

$$
\begin{gathered}
b_{k} \geq 0, \quad k=1, \ldots, m, \quad \sum_{k=1}^{m}\left|b_{k}\right|<1, \\
\alpha_{2 q+1}>0, \quad \sum_{k=1}^{n} \beta_{k}+\alpha_{2 q+1} \neq 0, \quad \sum_{k=1}^{n} \beta_{k}(n+1)^{(2 q+1) /(2 q+2)}<\alpha_{2 q+1} .
\end{gathered}
$$

\section{Proofs of theorems}

In this section, we prove that main theorems are presented in Section 2. We define the linear operator $L: D(L) \subseteq X \rightarrow Y$ and the nonlinear operator $N: X \rightarrow Y$ by

$$
L x(t)=\left(\begin{array}{c}
x^{\prime \prime}(t)+\alpha x^{\prime}(t)+\beta x(t) \\
\Delta x\left(t_{1}\right) \\
\vdots \\
\Delta x\left(t_{m}\right) \\
\Delta x^{\prime}\left(t_{1}\right) \\
\vdots \\
\Delta x^{\prime}\left(t_{m}\right)
\end{array}\right) \text { for } x \in D(L)
$$


where

$$
\begin{aligned}
& D(L)=\left\{\begin{array}{l|l}
u:[0, T] \longrightarrow R & \begin{array}{l}
u \in X \text { is differentiable in }\left(t_{k}, t_{k+1}\right)(k=0,1, \ldots, m), \\
\text { there exist the limits } \lim _{t \rightarrow t_{k}^{+}} x^{\prime}(t), \\
\lim _{t \rightarrow t_{k}^{-}} x^{\prime}(t)=x^{\prime}\left(t_{k}\right)(k=0,1, \ldots, m), \\
\lim _{t \rightarrow 0^{+}} x^{\prime}(t)=x^{\prime}(0), \lim _{t \rightarrow T^{-}} x^{\prime}(t)=x^{\prime}(T), \\
x^{\prime} \in X \text { there exist the limits } \lim _{t \rightarrow t_{k}^{+}} x^{\prime \prime}(t), \\
\lim _{t \rightarrow t_{k}^{-}} x^{\prime \prime}(t)=x^{\prime \prime}\left(t_{k}\right)(k=0,1, \ldots, m), \\
\lim _{t \rightarrow 0^{+}} x^{\prime \prime}(t)=x^{\prime \prime}(0), \lim _{t \rightarrow T^{-}} x^{\prime \prime}(t)=x^{\prime \prime}(T), \\
x^{\prime \prime} \in X,
\end{array}
\end{array},\right. \\
& N x(t)=\left(\begin{array}{c}
f\left(t, x(t), x\left(\alpha_{1}(t)\right), \ldots, x\left(\alpha_{n}(t)\right)\right) \\
I_{1}\left(x\left(t_{1}\right), x^{\prime}\left(t_{1}\right)\right) \\
\vdots \\
I_{m}\left(x\left(t_{m}\right), x^{\prime}\left(t_{m}\right)\right) \\
J_{1}\left(x\left(t_{1}\right), x^{\prime}\left(t_{1}\right)\right) \\
\vdots \\
J_{m}\left(x\left(t_{m}\right), x^{\prime}\left(t_{m}\right)\right)
\end{array}\right) \text { for } x \in X .
\end{aligned}
$$

Lemma 3.1. Suppose $\alpha \neq 0, \beta \neq 0$, or $\alpha=0$ and $\beta<0, f:[0, T] \times R^{n+1} \rightarrow R$ is an impulsive Caratheodory function, $I_{k}, J_{k}: R^{2} \rightarrow R$ are continuous. Then, the following results hold.

(i) $\operatorname{Ker} L=\{0\}$.

(ii) $L$ is a Fredholm operator of index zero.

(iii) $N$ is L-compact on $\bar{\Omega}$ with $\Omega$ being open and bounded.

Lemma 3.2 ([15]). Let $X$ and $Y$ be Banach spaces. Suppose $L: D(L) \subset X \rightarrow Y$ is a Fredholm operator of index zero with $\operatorname{Ker} L=\{0\}, N: X \rightarrow Y$ is L-compact on any open-bounded subset of $X$. If $0 \in \Omega \subset X$ is an open-bounded subset and $L x \neq \lambda N x$ for all $x \in D(L) \cap \partial \Omega$ and $\lambda \in[0,1]$, then there exists at least one $x \in \Omega$ such that $L x=N x$.

Lemma 3.3. Suppose $\beta<0$ and $\alpha \geq 0$ and $\left(A_{1}\right),\left(A_{2}\right),\left(A_{3}\right)$, and $(C)$ hold. Let $\Omega_{1}=\{x \in D(L)$ : $L x=\lambda N x, \exists \lambda \in(0,1)\}$. Then, $\Omega_{1}$ is bounded if (2.11) holds.

Proof. Suppose $x \in \Omega_{1}$, then

$$
\begin{gathered}
x^{\prime \prime}(t)+\alpha x^{\prime}(t)+\beta x(t)=\lambda f\left(t, x(t), x\left(\alpha_{1}(t)\right), \ldots, x\left(\alpha_{n}(t)\right)\right), \quad \text { a.e. } t \in[0, T], \\
\Delta x\left(t_{k}\right)=\lambda I_{k}\left(x\left(t_{k}\right), x^{\prime}\left(t_{k}\right)\right), \quad k=1, \ldots, m, \\
\Delta x^{\prime}\left(t_{k}\right)=\lambda J_{k}\left(x\left(t_{k}\right), x^{\prime}\left(t_{k}\right)\right), \quad k=1, \ldots, m, \\
x(0)=x(T), \quad x^{\prime}(0)=x^{\prime}(T) .
\end{gathered}
$$


Step 1. Prove that there exists a constant $M_{1}>0$ so that $\int_{0}^{T}|x(s)|^{q+1} d s \leq M_{1}$ for each $x \in \Omega_{1}$. Multiplying both sides of the first equation of (3.3) by $x(t)$, integrating it from 0 to $T$, we get from $(C)$ that

$$
\begin{gathered}
x^{\prime}(T) x(T)-x^{\prime}(0) x(0)-\sum_{k=1}^{m}\left[x^{\prime}\left(t_{k}^{+}\right) x\left(t_{k}^{+}\right)-x^{\prime}\left(t_{k}\right) x\left(t_{k}\right)\right]-\int_{0}^{T}\left[x^{\prime}(s)\right]^{2} d s \\
+\frac{\alpha}{2}\left[(x(T))^{2}-(x(0))^{2}\right]-\frac{\alpha}{2} \sum_{k=1}^{m}\left[\left(x\left(t_{k}^{+}\right)\right)^{2}-\left(x\left(t_{k}\right)\right)^{2}\right]+\beta \int_{0}^{T}|x(t)|^{2} d t \\
=\lambda \int_{0}^{T} f\left(s, x(s), x\left(\alpha_{1}(s)\right), \ldots, x\left(\alpha_{n}(s)\right)\right) x(s) d s \\
=\lambda\left(\int_{0}^{T} h\left(s, x(s), x\left(\alpha_{1}(s)\right), \ldots, x\left(\alpha_{n}(s)\right)\right) x(s) d s+\int_{0}^{T} g_{0}(s, x(s)) x(s) d s\right. \\
\left.\quad+\sum_{i=1}^{n} \int_{0}^{T} g_{i}\left(s, x\left(\alpha_{i}(s)\right)\right) x(s) d s+\int_{0}^{T} r(s) x(s) d s\right) .
\end{gathered}
$$

It follows from $\left(A_{1}\right)$ that

$$
\begin{aligned}
\sum_{k=1}^{m}\left[\left(x\left(t_{k}^{+}\right)\right)^{2}-\left(x\left(t_{k}\right)\right)^{2}\right] & =\sum_{k=1}^{m}\left(x\left(t_{k}^{+}\right)-x\left(t_{k}\right)\right)\left(x\left(t_{k}^{+}\right)+x\left(t_{k}\right)\right) \\
& =\sum_{k=1}^{m} \Delta x\left(t_{k}\right)\left(2 x\left(t_{k}\right)+\Delta x\left(t_{k}^{-}\right)\right) \\
& =\lambda \sum_{k=1}^{m} I_{k}\left(x\left(t_{k}\right), x^{\prime}\left(t_{k}\right)\right)\left(2 x\left(t_{k}\right)+\lambda I_{k}\left(x\left(t_{k}\right), x^{\prime}\left(t_{k}\right)\right)\right) \\
& \geq 2 \lambda \sum_{k=1}^{m} I_{k}\left(x\left(t_{k}\right), x^{\prime}\left(t_{k}\right)\right) x\left(t_{k}\right) \geq 0 .
\end{aligned}
$$

On the other hand, $\left(A_{2}\right)$ implies that

$$
\begin{aligned}
\sum_{k=1}^{m}\left(x^{\prime}\left(t_{k}^{+}\right) x\left(t_{k}^{+}\right)-x^{\prime}\left(t_{k}\right) x\left(t_{k}\right)\right)= & \left.\sum_{k=1}^{m}\left[x^{\prime}\left(t_{k}^{+}\right)\left(x\left(t_{k}^{+}\right)-x\left(t_{k}\right)\right)+\left(x^{\prime}\left(t_{k}^{+}\right)-x^{\prime}\left(t_{k}\right)\right) x\left(t_{k}\right)\right)\right] \\
= & \lambda \sum_{k=1}^{m}\left(x^{\prime}\left(t_{k}\right) I_{k}\left(x\left(t_{k}\right), x^{\prime}\left(t_{k}\right)\right)+x\left(t_{k}\right) J_{k}\left(x\left(t_{k}\right), x^{\prime}\left(t_{k}\right)\right)\right) \\
& +\lambda^{2} \sum_{k=1}^{m} I_{k}\left(x\left(t_{k}\right), x^{\prime}\left(t_{k}\right)\right) J_{k}\left(x\left(t_{k}\right), x^{\prime}\left(t_{k}\right)\right) \\
\geq & 0 .
\end{aligned}
$$

We get

$$
\begin{gathered}
\int_{0}^{T} h\left(s, x(s), x\left(\alpha_{1}(s)\right), \ldots, x\left(\alpha_{n}(s)\right)\right) x(s) d s+\int_{0}^{T} g_{0}(s, x(s)) x(s) d s \\
+\sum_{i=1}^{n} \int_{0}^{T} g_{i}\left(s, x\left(\alpha_{i}(s)\right)\right) x(s) d s+\int_{0}^{T} r(s) x(s) d s \leq 0 .
\end{gathered}
$$


It follows from $(C)$ that

$$
\begin{aligned}
\theta \int_{0}^{T}|x(s)|^{q+1} d s \leq & -\int_{0}^{T} g_{0}(s, x(s)) x(s) d s-\sum_{i=1}^{n} \int_{0}^{T} g_{i}\left(s, x\left(\alpha_{i}(s)\right)\right) x(s) d s \\
& +\int_{0}^{T} r(s) x(s) d s+\beta \int_{0}^{T}|x(t)|^{2} d t \\
\leq & \int_{0}^{T}\left|g_{0}(s, x(s))\right||x(s)| d s+\sum_{i=1}^{n} \int_{0}^{T}\left|g_{i}\left(s, x\left(\alpha_{i}(s)\right)\right)\right||x(s)| d s \\
& +\int_{0}^{T}|r(s)||x(s)| d s .
\end{aligned}
$$

Let $\epsilon>0$ satisfy

$$
\theta>\left(r_{0}+\epsilon\right)+\sum_{k=1}^{n}\left(r_{k}+\epsilon\right)\left\|\beta_{k}^{\prime}\right\|_{\infty}^{q /(q+1)} .
$$

For such $\epsilon>0$, there exists $\delta>0$ such that for every $i=0,1, \ldots, n$,

$$
\left|g_{i}(t, x)\right|<\left(r_{i}+\epsilon\right)|x|^{q} \text { for a.e. } t \in[0, T] \text { and all } x \text { such that }|x|>\delta .
$$

Let, for $i=1, \ldots, n, \Delta_{1, i}=\left\{t: t \in[0, T],\left|x\left(\alpha_{i}(t)\right)\right| \leq \delta\right\}, \Delta_{2, i}=\left\{t: t \in[0, T],\left|x\left(\alpha_{i}(t)\right)\right|>\delta\right\}$, $g_{\delta, i}=\max _{t \in[0, T],|x| \leq \delta}\left|g_{i}(t, x)\right|$, and $\Delta_{1}=\{t \in[0, T],|x(t)| \leq \delta\}, \Delta_{2}=\{t \in[0, T],|x(t)|>\delta\}$, and $\delta^{\prime}=\max \left\{g_{\delta, k}: k=0, \ldots, n\right\}$. Then, we get

$$
\begin{aligned}
\theta \int_{0}^{T}|x(s)|^{q+1} d s & \\
= & \int_{\Delta_{1}}\left|g_{0}(s, x(s))\right||x(s)| d s+\int_{\Delta_{2}}\left|g_{0}(s, x(s))\right||x(s)| d s+\sum_{i=1}^{n} \int_{\Delta_{1, i}}\left|g_{i}\left(s, x\left(\alpha_{i}(s)\right)\right)\right||x(s)| d s \\
& +\sum_{i=1}^{n} \int_{\Delta_{2, i}}\left|g_{i}\left(s, x\left(\alpha_{i}(s)\right)\right)\right||x(s)| d s+\int_{0}^{T}|r(s)||x(s)| d s \\
\leq & \left(r_{0}+\epsilon\right) \int_{0}^{T}|x(s)|^{q+1} d s+\sum_{k=1}^{n}\left(r_{k}+\epsilon\right) \int_{0}^{T}\left|x\left(\alpha_{k}(s)\right)\right|^{q}|x(s)| d s \\
& +\int_{0}^{T}|r(s)||x(s)| d s+g_{\delta, 0} \int_{0}^{T}|x(s)| d s+\sum_{k=1}^{n} g_{\delta, k} \int_{0}^{T}|x(s)| d s \\
\leq & \left(r_{0}+\epsilon\right) \int_{0}^{T}|x(s)|^{q+1} d s \\
& +\sum_{k=1}^{n}\left(r_{k}+\epsilon\right)\left(\int_{0}^{T}\left|x\left(\alpha_{k}(s)\right)\right|^{q+1} d s\right)^{q /(q+1)}\left(\int_{0}^{T}|x(s)|^{q+1} d s\right)^{1 /(q+1)} \\
& +\left(\int_{0}^{T}|r(s)|^{(q+1) / q} d s\right)^{q /(q+1)}\left(\int_{0}^{T}|x(s)|^{q+1} d s\right)^{1 /(q+1)}+(n+1) \delta^{\prime} \int_{0}^{T}|x(s)| d s \\
= & \left(r_{0}+\epsilon\right) \int_{0}^{T}|x(s)|^{q+1} d s+\left.\left.\sum_{k=1}^{n}\left(r_{k}+\epsilon\right)\left|\int_{\alpha_{k}(0)}^{\alpha_{k}(T)}\right| x(u)\right|^{q+1}\left|\beta_{k}^{\prime}(u)\right| d u\right|^{q /(q+1)}\left(\int_{0}^{T}|x(s)|^{q+1} d s\right)^{1 /(q+1)}
\end{aligned}
$$


X. Liu and Y. Liu

$$
\begin{aligned}
& +\left(\int_{0}^{T}|r(s)|^{(q+1) / q} d s\right)^{q /(q+1)}\left(\int_{0}^{T}|x(s)|^{q+1} d s\right)^{1 /(q+1)} \\
& +(n+1) \delta^{\prime} T^{q /(q+1)}\left(\int_{0}^{T}|x(s)|^{q+1} d s\right)^{1 /(q+1)} \\
& \leq\left(r_{0}+\epsilon\right) \int_{0}^{T}|x(s)|^{q+1} d s \\
& +\sum_{k=1}^{n}\left(r_{k}+\epsilon\right)\left\|\beta_{k}^{\prime}\right\|^{q /(q+1)}\left(\int_{0}^{T}|x(u)|^{1+q} d u\right)^{q /(q+1)}\left(\int_{0}^{T}|x(s)|^{q+1} d s\right)^{1 /(q+1)} \\
& +\left(\int_{0}^{T}|r(s)|^{(q+1) / q} d s\right)^{q /(q+1)}\left(\int_{0}^{T}|x(s)|^{q+1} d s\right)^{1 /(q+1)} \\
& +(n+1) \delta^{\prime} T^{q /(q+1)}\left(\int_{0}^{T}|x(s)|^{q+1} d s\right)^{1 /(q+1)} \\
& =\left(\left(r_{0}+\epsilon\right)+\sum_{k=1}^{n}\left(r_{k}+\epsilon\right)\left\|\beta_{k}^{\prime}\right\|^{q /(q+1)}\right) \int_{0}^{T}|x(s)|^{q+1} d s \\
& +\left(\int_{0}^{T}|r(s)|^{(q+1) / q} d s\right)^{q /(q+1)}\left(\int_{0}^{T}|x(s)|^{q+1} d s\right)^{1 /(q+1)} \\
& +(n+1) \delta^{\prime} T^{q /(q+1)}\left(\int_{0}^{T}|x(s)|^{q+1} d s\right)^{1 /(q+1)} .
\end{aligned}
$$

Then, (3.9) implies that there exists a constant $M_{1}>0$ such that $\int_{0}^{T}|x(s)|^{q+1} d s \leq M_{1}$.

Step 2. Prove that there exists a constant $M_{2}>0$ such that $\|x\|_{\infty} \leq M_{2}$ for each $x \in \Omega_{1}$.

It follows from Step 1 that there exists $\xi \in[0, T]$ such that $|x(\xi)| \leq\left(M_{1} / T\right)^{1 /(q+1)}$.

Multiplying both sides of the first equation of (3.3) by $x(t)$, integrating it from 0 to $T$, we get, using $\left(A_{1}\right),\left(A_{2}\right)$, and $(C)$,

$$
\begin{aligned}
\int_{0}^{T}\left[x^{\prime}(s)\right]^{2} d s= & -\frac{1}{2} \sum_{k=1}^{m}\left[\left(x\left(t_{k}^{+}\right)\right)^{2}-\left(x\left(t_{k}\right)\right)^{2}\right]-\sum_{k=1}^{m}\left[x^{\prime}\left(t_{k}^{+}\right) x\left(t_{k}^{+}\right)-x^{\prime}\left(t_{k}\right) x\left(t_{k}\right)\right] \\
& -\lambda \int_{0}^{T} f\left(s, x(s), x\left(\alpha_{1}(s)\right), \ldots, x\left(\alpha_{n}(s)\right)\right) x(s) d s+\lambda \beta \int_{0}^{T}|x(t)|^{2} d t \\
\leq & -\lambda \int_{0}^{T} f\left(s, x(s), x\left(\alpha_{1}(s)\right), \ldots, x\left(\alpha_{n}(s)\right)\right) x(s) d s+\lambda \beta \int_{0}^{T}|x(t)|^{2} d t \\
\leq & -\lambda\left(\int_{0}^{T} h\left(s, x(s), x\left(\alpha_{1}(s)\right), \ldots, x\left(\alpha_{n}(s)\right)\right) x(s) d s+\int_{0}^{T} g_{0}(s, x(s)) x(s) d s\right. \\
& \left.\quad+\sum_{i=1}^{n} \int_{0}^{T} g_{i}\left(s, x\left(\alpha_{i}(s)\right)\right) x(s) d s+\int_{0}^{T} r(s) x(s) d s\right)+\lambda \beta \int_{0}^{T}|x(t)|^{2} d t
\end{aligned}
$$




$$
\begin{aligned}
& \leq-\lambda \int_{0}^{T} g_{0}(s, x(s)) x(s) d s-\lambda \sum_{i=1}^{n} \int_{0}^{T} g_{i}\left(s, x\left(\alpha_{i}(s)\right)\right) x(s) d s-\lambda \int_{0}^{T} r(s) x(s) d s \\
& \leq \int_{0}^{T}\left|g_{0}(s, x(s))\right||x(s)| d s+\sum_{i=1}^{n} \int_{0}^{T}\left|g_{i}\left(s, x\left(\alpha_{i}(s)\right)\right)\right||x(s)| d s+\int_{0}^{T}|r(s)||x(s)| d s \\
& \leq\left[\left(\left(r_{0}+\epsilon\right)+\sum_{k=1}^{n}\left(r_{k}+\epsilon\right)\left\|\beta_{k}^{\prime}\right\|_{\infty}^{q /(1+q)}\right) \int_{0}^{T}|x(s)|^{q+1} d s\right. \\
& \left.\quad+\left(\int_{0}^{T}|r(s)|^{(q+1) / q} d s\right)^{q /(q+1)}\left(\int_{0}^{T}|x(s)|^{q+1} d s\right)^{1 /(q+1)}\right] \\
& \quad+(n+1) \delta^{\prime} T^{q /(q+1)}\left(\int_{0}^{T}|x(s)|^{q+1} d s\right)^{1 /(q+1)} .
\end{aligned}
$$

Then,

$$
\begin{aligned}
\int_{0}^{T}\left[x^{\prime}(s)\right]^{2} d s \leq & {\left[\left(\left(r_{0}+\epsilon\right)+\sum_{k=1}^{n}\left(r_{k}+\epsilon\right)\left\|\beta_{k}^{\prime}\right\|_{\infty}^{q /(1+q)}\right) M_{1}+\left(\int_{0}^{T}|r(s)|^{(q+1) / q} d s\right)^{q /(q+1)} M_{1}^{1 /(q+1)}\right] } \\
& +(n+1) \delta^{\prime} T^{q /(q+1)} M_{1}^{1 /(q+1)} \\
= & : M_{2} .
\end{aligned}
$$

Due to $\left(A_{3}\right)$, one sees that

$$
\begin{aligned}
|x(t)| & =\left\{\begin{array}{l}
\left|x(\xi)+\lambda \sum_{\xi \leq t_{k}<t} I_{k}\left(x\left(t_{k}\right), x^{\prime}\left(t_{k}\right)\right)+\int_{\xi}^{t} x^{\prime}(s) d s\right| \text { if } t \geq \xi, \\
\left|x(\xi)-\lambda \sum_{t \leq t_{k}<\xi} I_{k}\left(x\left(t_{k}\right), x^{\prime}\left(t_{k}\right)\right)-\int_{t}^{\xi} x^{\prime}(s) d s\right| \text { if } t<\xi,
\end{array}\right. \\
& \leq\left(\frac{M_{1}}{T}\right)^{1 /(q+1)}+\sum_{k=1}^{m} \theta_{k}\|x\|+\int_{0}^{T}\left|x^{\prime}(s)\right| d s \\
& \leq\left(\frac{M_{1}}{T}\right)^{1 /(q+1)}+\sum_{k=1}^{m} \theta_{k}\|x\|+T^{1 / 2}\left(\int_{0}^{T}\left|x^{\prime}(s)\right|^{2} d s\right)^{1 / 2} \\
& \leq\left(\frac{M_{1}}{T}\right)^{1 /(q+1)}+\sum_{k=1}^{m} \theta_{k}\|x\|+T^{1 / 2} M_{2}^{1 / 2} .
\end{aligned}
$$

It follows from $\left(A_{3}\right)$ that

$$
\|x\|_{\infty} \leq \frac{1}{1-\sum_{k=1}^{m} \theta_{k}}\left(\left(\frac{M_{1}}{T}\right)^{1 /(q+1)}+T^{1 / 2} M_{2}^{1 / 2}\right) .
$$

It follows that $\Omega_{1}$ is bounded. This completes the proof of Lemma 3.3.

Lemma 3.4. Suppose $\beta<0$ and $\alpha>0$ and $\left(A_{1}^{\prime}\right),\left(A_{2}\right),\left(A_{3}\right)$, and $(C)$ hold. Let $\Omega_{1}=\{x \in D(L)$ : $L x=\lambda N x, \exists \lambda \in(0,1)\}$. Then, $\Omega_{1}$ is bounded if (2.11) holds. 
Proof. The proof is similar to that of Lemma 3.3.

Lemma 3.5. Suppose $\beta>0$ and $\alpha>0$ and $\left(A_{1}^{\prime}\right),\left(A_{2}^{\prime}\right),\left(A_{3}\right)$, and $\left(C^{\prime}\right)$ hold. Let $\Omega_{1}=\{x \in D(L)$ : $L x=\lambda N x, \exists \lambda \in(0,1)\}$. Then, $\Omega_{1}$ is bounded if (2.11) holds.

Proof. Suppose $x \in \Omega_{1}$, then we get (3.3).

Step 1. Prove that there exists a constant $M_{1}>0$ so that $\int_{0}^{T}|x(s)|^{q+1} d s \leq M_{1}$ for each $x \in \Omega_{1}$. Multiplying both sides of the first equation of (3.3) by $x(t)$, integrating it from 0 to $T$, we get from $\left(C^{\prime}\right)$

$$
\begin{gathered}
x^{\prime}(T) x(T)-x^{\prime}(0) x(0)-\sum_{k=1}^{m}\left[x^{\prime}\left(t_{k}^{+}\right) x\left(t_{k}^{+}\right)-x^{\prime}\left(t_{k}\right) x\left(t_{k}\right)\right]-\int_{0}^{T}\left[x^{\prime}(s)\right]^{2} d s \\
+\frac{\alpha}{2}\left[(x(T))^{2}-(x(0))^{2}\right]-\frac{\alpha}{2} \sum_{k=1}^{m}\left[\left(x\left(t_{k}^{+}\right)\right)^{2}-\left(x\left(t_{k}\right)\right)^{2}\right]+\beta \int_{0}^{T}|x(t)|^{2} d t \\
=\lambda\left(\int_{0}^{T} h\left(s, x(s), x\left(\alpha_{1}(s)\right), \ldots, x\left(\alpha_{n}(s)\right)\right) x(s) d s+\int_{0}^{T} g_{0}(s, x(s)) x(s) d s\right. \\
+\sum_{i=1}^{n} \int_{0}^{T} g_{i}\left(s, x\left(\alpha_{i}(s)\right) x(s) d s+\int_{0}^{T} r(s) x(s) d s\right) .
\end{gathered}
$$

It follows from $\left(A_{1}^{\prime}\right)$ that

$$
\begin{aligned}
\sum_{k=1}^{m}\left[\left(x\left(t_{k}^{+}\right)\right)^{2}-\left(x\left(t_{k}\right)\right)^{2}\right] & =\sum_{k=1}^{m}\left(x\left(t_{k}^{+}\right)-x\left(t_{k}\right)\right)\left(x\left(t_{k}^{+}\right)+x\left(t_{k}\right)\right) \\
& =\sum_{k=1}^{m} \Delta x\left(t_{k}\right)\left(2 x\left(t_{k}\right)+\Delta x\left(t_{k}^{-}\right)\right) \\
& =\lambda \sum_{k=1}^{m} I_{k}\left(x\left(t_{k}\right), x^{\prime}\left(t_{k}\right)\right)\left(2 x\left(t_{k}\right)+\lambda I_{k}\left(x\left(t_{k}\right), x^{\prime}\left(t_{k}\right)\right)\right) \\
& \leq \lambda \sum_{k=1}^{m} I_{k}\left(x\left(t_{k}\right), x^{\prime}\left(t_{k}\right)\right)\left(2 x\left(t_{k}\right)+I_{k}\left(x\left(t_{k}\right), x^{\prime}\left(t_{k}\right)\right)\right) \\
& \leq 0 .
\end{aligned}
$$

On the other hand, $\left(A_{2}^{\prime}\right)$ implies that

$$
\begin{aligned}
\sum_{k=1}^{m}\left(x^{\prime}\left(t_{k}^{+}\right) x\left(t_{k}^{+}\right)-x^{\prime}\left(t_{k}\right) x\left(t_{k}\right)\right)= & \left.\sum_{k=1}^{m}\left[x^{\prime}\left(t_{k}^{+}\right)\left(x\left(t_{k}^{+}\right)-x\left(t_{k}\right)\right)+\left(x^{\prime}\left(t_{k}^{+}\right)-x^{\prime}\left(t_{k}\right)\right) x\left(t_{k}\right)\right)\right] \\
= & \lambda \sum_{k=1}^{m}\left(x^{\prime}\left(t_{k}\right) I_{k}\left(x\left(t_{k}\right), x^{\prime}\left(t_{k}\right)\right)+x\left(t_{k}\right) J_{k}\left(x\left(t_{k}\right), x^{\prime}\left(t_{k}\right)\right)\right) \\
& +\lambda^{2} \sum_{k=1}^{m} I_{k}\left(x\left(t_{k}\right), x^{\prime}\left(t_{k}\right)\right) J_{k}\left(x\left(t_{k}\right), x^{\prime}\left(t_{k}\right)\right)
\end{aligned}
$$

$\leq 0$. 
We get

$$
\begin{gathered}
\lambda\left(\int_{0}^{T} h\left(s, x(s), x\left(\alpha_{1}(s)\right), \ldots, x\left(\alpha_{n}(s)\right)\right) x(s) d s+\int_{0}^{T} g_{0}(s, x(s)) x(s) d s\right. \\
+\sum_{i=1}^{n} \int_{0}^{T} g_{i}\left(s, x\left(\alpha_{i}(s)\right) x(s) d s+\int_{0}^{T} r(s) x(s) d s\right) \geq 0 .
\end{gathered}
$$

It follows from $\left(C^{\prime}\right)$ that

$$
\begin{aligned}
\theta \int_{0}^{T}|x(s)|^{q+1} d s \leq & \int_{0}^{T} g_{0}(s, x(s)) x(s) d s-\sum_{i=1}^{n} \int_{0}^{T} g_{i}\left(s, x\left(\alpha_{i}(s)\right) x(s) d s\right. \\
& +\int_{0}^{T} r(s) x(s) d s+\beta \int_{0}^{T}|x(t)|^{2} d t \\
\leq & \int_{0}^{T}\left|g_{0}(s, x(s))\right||x(s)| d s+\sum_{i=1}^{n} \int_{0}^{T} \mid g_{i}\left(s, x\left(\alpha_{i}(s)\right)|| x(s) \mid d s\right. \\
& +\int_{0}^{T}|r(s)||x(s)| d s .
\end{aligned}
$$

The remainder of the proof is similar to that of the corresponding part of the proof of Lemma 3.3.

Lemma 3.6. Suppose $\beta>0$ and $\alpha<0$ and $\left(A_{1}\right),\left(A_{2}\right),\left(A_{3}\right)$, and $(C)$ hold. Let $\Omega_{1}=\{x \in D(L)$ : $L x=\lambda N x, \exists \lambda \in(0,1)\}$. Then, $\Omega_{1}$ is bounded if (2.11) holds.

Proof. The proof is similar to that of Lemma 3.5.

Proof of Theorem 2.1. It is easy to show that $L: D(L) \subset X \rightarrow Y$ is a Fredholm operator of index zero with $\operatorname{Ker} L=\{0\}, N: X \rightarrow Y$ is $L$-compact on any open-bounded subset of $X$. Let $\Omega$ satisfy $0 \in \overline{\Omega_{1}} \subset \Omega \subset X$ which is a open-bounded subset, where $\Omega_{1}$ is given in Lemma 3.3. It follows from Lemma 3.3 that $L x \neq \lambda N x$ for all $x \in D(L) \cap \partial \Omega$ and $\lambda \in[0,1]$, then there exists at least one $x \in \Omega$ such that $L x=N x$. Hence, $x$ is a solution of PBVP (1.13).

Proof of Theorem 2.2. Following Lemma 3.4, the proof is similar to that of Theorem 2.1.

Proof of Theorem 2.3. Following Lemma 3.5, the proof is similar to that of Theorem 2.1.

Proof of Theorem 2.4. Following Lemma 3.6, the proof is similar to that of Theorem 2.1.

\section{Acknowledgments}

The authors are very grateful to the referee for his or her suggestions to rearrange the paper details. The first author is supported by Science Foundation of Educational Committee of Hunan province (08C794), and both authors are supported by Natural Science Foundation of Hunan province, China (no. 06JJ5008) and Natural Science Foundation of Guangdong province (no. 7004569 ). 


\section{References}

[1] T. R. Ding, "Nonlinear oscillations at a point of resonance," Scientia Sinica Series A, vol. 25, no. 9, pp. 918-931, 1982.

[2] W. Ding, M. Han, and J. Yan, "Periodic boundary value problems for the second order functional differential equations," Journal of Mathematical Analysis and Applications, vol. 298, no. 1, pp. 341-351, 2004.

[3] D. Jiang, J. J. Nieto, and W. Zuo, "On monotone method for first and second order periodic boundary value problems and periodic solutions of functional differential equations," Journal of Mathematical Analysis and Applications, vol. 289, no. 2, pp. 691-699, 2004.

[4] J. J. Nieto and R. Rodríguez-López, "Existence and approximation of solutions for nonlinear functional differential equations with periodic boundary value conditions," Computers $\mathcal{E}$ Mathematics with Applications, vol. 40, no. 4-5, pp. 433-442, 2000.

[5] J. J. Nieto and R. Rodríguez-López, "Remarks on periodic boundary value problems for functional differential equations," Journal of Computational and Applied Mathematics, vol. 158, no. 2, pp. 339-353, 2003.

[6] D. Jiang and J. Wei, "Monotone method for first- and second-order periodic boundary value problems and periodic solutions of functional differential equations," Nonlinear Analysis: Theory, Methods $\mathcal{E}$ Applications, vol. 50, no. 7, pp. 885-898, 2002.

[7] J. J. Nieto and N. Alvarez-Noriega, "Periodic boundary value problems for nonlinear first order ordinary differential equations," Acta Mathematica Hungarica, vol. 71, no. 1-2, pp. 49-58, 1996.

[8] S. Ma and J. Wu, "A small twist theorem and boundedness of solutions for semilinear Duffing equations at resonance," Nonlinear Analysis: Theory, Methods \& Applications, vol. 67, no. 1, pp. 200237, 2007.

[9] R. Liang and J. Shen, "Periodic boundary value problem for second-order impulsive functional differential equations," Applied Mathematics and Computation, vol. 193, no. 2, pp. 560-571, 2007.

[10] S. Ma, Z. Wang, and J. Yu, "Coincidence degree and periodic solutions of Duffing equations," Nonlinear Analysis: Theory, Methods \& Applications, vol. 34, no. 3, pp. 443-460, 1998.

[11] L. Peng, "Existence and uniqueness of periodic solutions for a kind of Duffing equation with two deviating arguments," Mathematical and Computer Modelling, vol. 45, no. 3-4, pp. 378-386, 2007.

[12] A. Cabada, "The method of lower and upper solutions for second, third, fourth, and higher order boundary value problems," Journal of Mathematical Analysis and Applications, vol. 185, no. 2, pp. 302320, 1994.

[13] P. Omari and M. Trombetta, "Remarks on the lower and upper solutions method for second- and third-order periodic boundary value problems," Applied Mathematics and Computation, vol. 50, no. 1, pp. 1-21, 1992.

[14] I. Kiguradze and S. Staněk, "On periodic boundary value problem for the equation $u^{\prime \prime}=f\left(t, u, u^{\prime}\right)$ with one-sided growth restrictions on $f$," Nonlinear Analysis: Theory, Methods $\mathcal{E}$ Applications, vol. 48, no. 7, pp. 1065-1075, 2002.

[15] R. E. Gaines and J. L. Mawhin, Coincidence Degree, and Nonlinear Differential Equations, Lecture Notes in Mathematics, Vol. 568, Springer, Berlin, Germany, 1977.

[16] C. P. Gupta, J. J. Nieto, and L. Sanchez, "Periodic solutions of some Lienard and Duffing equations," Journal of Mathematical Analysis and Applications, vol. 140, no. 1, pp. 67-82, 1989.

[17] J. J. Nieto and R. Rodríguez-López, "Green's function for second-order periodic boundary value problems with piecewise constant arguments," Journal of Mathematical Analysis and Applications, vol. 304, no. 1, pp. 33-57, 2005.

[18] Z. Zhang and J. Wang, "On existence and multiplicity of positive solutions to periodic boundary value problems for singular nonlinear second order differential equations," Journal of Mathematical Analysis and Applications, vol. 281, no. 1, pp. 99-107, 2003.

[19] D. Jiang, "On the existence of positive solutions to second order periodic BVPs," Acta Mathematica Sinica, vol. 18, no. 1, pp. 31-35, 1989.

[20] J.-M. Belley and M. Virgilio, "Periodic Duffing delay equations with state dependent impulses," Journal of Mathematical Analysis and Applications, vol. 306, no. 2, pp. 646-662, 2005.

[21] Z. Wang, "Multiplicity of periodic solutions of semilinear Duffing's equation at resonance," Journal of Mathematical Analysis and Applications, vol. 237, no. 1, pp. 166-187, 1999. 
[22] H. Wang and H. Chen, "Boundary value problem for second-order impulsive functional differential equations," Applied Mathematics and Computation, vol. 191, no. 2, pp. 582-591, 2007.

[23] X. Yang and J. Shen, "Periodic boundary value problems for second-order impulsive integrodifferential equations," Journal of Computational and Applied Mathematics, vol. 209, no. 2, pp. 176-186, 2007.

[24] Y. Liu and W. Ge, "On the positive periodic solutions of the nonlinear Duffing equations with delay and variable coefficients," Bulletin of the Belgian Mathematical Society. Simon Stevin, vol. 11, no. 3, pp. 441-455, 2004. 